\title{
Le peuple comme « multitude de créatures rationnelles »
}

\author{
La réponse de Sidney à Hobbes*
}

\author{
Christopher Hamel
}

Université libre de Bruxelles

\begin{abstract}
C'est une maxime générale que celui qui a établi peut aussi détruire, cujus est instituere, ejus est abrogare, surtout si cet établissement a été fait non seulement par lui, mais encore pour lui. Si donc le peuple (the multitude) établit une certaine forme de gouvernement, il est aussi en droit de l'abolir (the multitude may abrogate); et il n'y a que ce peuple (and they themselves), ou ceux qui lui succèdent dans ce droit qui soient capables, et à qui il appartient de juger si ceux qui les gouvernent le font conformément à ce qu'on s'était proposé en instituant cette espèce de gouvernement ${ }^{1}$.
\end{abstract}

\footnotetext{
* Je remercie chaleureusement Louis Carré et Thomas Berns pour leur relecture attentive et leurs suggestions pertinentes.

1. Algernon Sidney, Discours sur le gouvernement, trad. P. A. Samson, La Haye, Louis et Henri van Dole, 1702, (3 vol.), ch. I, sec. 6, vol. 1, p. 38. Je cite désormais (en traduisant) l'édition de T. West, Discourses concerning government, Indianapolis, Liberty Fund, 1996, abrégée ainsi : D, suivi du numéro du chapitre (en chiffres romains) et du numéro de la section (en chiffres arabes).
} 
Visiblement frappé par cet extrait des Discours sur le gouvernement d'Algernon Sidney, ouvrage posthume édité en 1698 et traduit en français par P. A. Samson en 1702, Rousseau recopie sur un cahier la maxime latine au cours de sa lecture de l'ouvrage 2 . À lire Sidney dans cette traduction, on conçoit fort bien pourquoi l'auteur du Contrat social, dans le passage de la sixième des Lettres écrites de la montagne où il cherche à défendre son livre condamné, 1. fait de la question très hobbesienne : «Qu'est-ce qui fait qu'un État est un? », le point de départ théorique du Contrat social; 2 . accuse néanmoins les «principes» de Hobbes d'être «destructifs de tout gouvernement républicain » et 3 . déclare sans ambages : au sujet « du droit naturel et politique », «Sidney pensait comme moi ${ }^{3}$ ». Ce dernier affirme en effet la souveraineté inaliénable du peuple, quand Hobbes soumet tout - sujets et lois - à la volonté du souverain.

Toutefois, en substituant le «peuple» singulier à la «multitude» plurielle, la traduction fautive de P. A. Samson a rendu invisible à Rousseau, qui ne lisait pas l'anglais, ce qui le rapprochant de Hobbes l'éloignait de Sidney: dans le développement dont est tiré l'extrait cité, l'agent qui établit et détruit est précisément ce que Hobbes et Rousseau opposent au peuple, à savoir une multitude.

Or réhabiliter la multitude pour penser le peuple comme agent politique est capital pour Sidney : c'est par ce moyen qu'il justifie le droit de résistance de l'individu au gouvernement tyrannique. Parce qu'elle reposait sur l'institution d'un pouvoir absolu censé contenir, tenir lieu ou représenter la volonté des particuliers, la solution de Hobbes à la question dont Rousseau hérite (qu'est-ce qui fait qu'un État est un ?) a suscité des oppositions, qui culminent probablement au dix-septième siècle dans plusieurs paragraphes du Second traité sur le gouvernement de John Locke, où celui-ci défend la thèse d'un droit de résistance du peuple entendu distributivement comme une collection de consciences interprétant pour elles-mêmes la loi de

2. Voir le Ms R 18 (ou 7842) de Rousseau, conservé à la Bibliothèque publique et universitaire de Neuchâtel. Voir Miriam Giargia, Disuguaglianza e virtù. Rousseau e il repubblicanesimo inglese, Milano, Edizioni Universitarie di Lettere Economia Diritto, 2008, pp. 50-57, qui cite tous les textes que Rousseau copie à la lecture de l'ouvrage de Sidney.

3. Jean-Jacques Rousseau, Lettres écrites de la montagne, VI, in Euvres complètes III, Paris, Gallimard, 1964, respectivement pp. 806, 811, 812. Sur l'unité du peuple, voir Contrat social, I, 5. 
la nature 4 . Si la thèse que défend Locke a une histoire - qui commence avec ceux qui, après et contre Hobbes, tentent de maintenir l'idée que la communauté morale d'individus qui est à l'origine du pouvoir politique ne disparaît pas une fois constituée l'autorité politique 5 - elle est aussi plus ou moins contemporaine de la version qu'en propose Sidney. Similaire à celle de Locke quant aux prémisses (individualistes) comme dans les effets (justification du droit individuel de résistance au gouvernement tyrannique) la position de Sidney a l'intérêt, pour justifier ce droit, d'utiliser le terme même sur lequel Hobbes en avait élaboré la réfutation.

Le souci de réfuter la légitimité de la résistance est à coup sûr l'une des toutes premières priorités de la «science civile » que Hobbes développe. Dès la préface du De Cive, il explique que l'erreur et l'ignorance causent nécessairement «conflits, querelles et meurtres » et que l'utilité de la science civile se mesure à l'aune des dommages qu'elle permet d'éviter. Or, s'il reconnaît qu'il existe de nombreuses autres «opinions non moins dangereuses », c'est la question de la légitimité de la résistance au pouvoir établi qui retient avant tout son intérêt : qu'il s'agisse de la théorie classique de la légitimité du tyrannicide, du droit de déposer un monarque, de la thèse monarchomaque du roi ministre d'un peuple qui lui est supérieur, du droit d'examen par la conscience de la légitimité des lois, ou de la version théologique du droit de résistance, Hobbes fait savoir que les réfuter est son «dessein 6 ». Le concept de multitude est un opérateur essentiel de cette réfutation?.

Nous verrons d'abord comment Sidney répond à l'accusation d'irrationalité en mettant la liberté rationnelle au

4. Voir John Locke, Second traité du gouvernement, trad. J.-F. Spitz et Ch. Lazzeri, Paris, PUF, 1994, §§ 241, 168, 21, 209, 212, 219.

5. Sur cette histoire, voir J.-F. Spitz, John Locke et les fondements de la liberté moderne, Paris, PUF, 2001 et J. H. Franklin, John Locke and the theory of sovereignity: mixed monarchy and the right of resistance in the political thought of the English Revolution, Cambridge, CUP, 1978. L'ouvrage de R. Ashcraft, Revolutionary Politics \& Locke's Two Treatises of Government, Princeton, PUP, 1986 (trad. fr. 1995), fournit l'indispensable reconstruction du débat idéologique auquel Locke et Sidney, entre autres, prennent part.

6. Thomas Hobbes, Du citoyen, trad. Ph. Crignon, Paris, GF, 2010, Préf., pp. 83, 89-90, 92 (désormais abrégé DC).

7. Les textes principaux sont Éléments de la loi naturelle et politique, trad. D. Weber, Paris, LGF, 2003, II, 1, 11, pp. 248-249 (désormais abrégé EL); DC, VI, 1, pp. 166-168, XII, 8, pp. 247-248 ; Léviathan, trad. F. Tricaud, Paris, Sirey, 1971, XVI, p. 177 (désormais abrégé L). 
cœur des finalités de l'association. Nous nous pencherons ensuite sur la manière dont Sidney refuse l'opposition construite par Hobbes entre peuple et multitude. Enfin, nous examinerons, du point de vue de Sidney, le caractère excessif de certaines conséquences de la thèse hobbesienne de l'unité du peuple, et montrerons qu'elles n'apparaissent plus nécessaires dans le cadre de l'individualisme moral défendu par Sidney.

\section{Le droit de résistance du peuple : une thèse contradictoire ?}

\section{Le désir de liberté, cause des guerres civiles}

La première occurrence politique du terme multitude dans le Léviathan intervient dans un passage du chapitre VIII où Hobbes décrit les gradations de la folie, laquelle est définie comme un excès passionnel. Si la folie d'un individu qui se prétend divinement «inspiré » ne se manifestera pas nécessairement de façon éclatante, il en va autrement, souligne Hobbes, d'une «multitude entière »: sa rage, elle, est bien visible. Dans une allusion évidente à la guerre civile anglaise qui fit vaciller la monarchie, il illustre son propos en invoquant l'absurdité d'une insurrection contre le pouvoir politique établi. Si en effet un individu inspiré est capable de «poursuivre ses meilleurs amis avec des vociférations, des coups et des pierres [...] de tels actes restent passablement en deçâ de ce que fera une multitude de ce genre. Car ils [i.e. les membres de cette multitude] vocifèreront et se battront contre et détruiront ceux par qui ils ont été protégés et défendus contre les torts toute leur vie durant 8 ».

Toutefois, Hobbes n'est en mesure de présenter comme irrationnelle une telle insurrection que parce qu'il entend les notions de «protection » et de « défense » des sujets en un sens qui n'aurait pas satisfaisait ceux qui, pour des motifs théologiques, politiques ou sociaux, étaient prêts à légitimer la résistance au pouvoir établi. En effet, si aucune des justifications de la résistance formulées dans le contexte de la guerre civile anglaise ne contestait que la protection et la paix fussent des biens recherchés dans l'association, nombre d'entre elles prétendaient en revanche que ces biens ne pouvaient pas être pensés indépendamment de la liberté. En soumettant le peuple à l'exercice d'une prérogative incontrôlable, le souverain violait

8. L, VIII, p. 71 (trad. modif.) ; voir aussi DC, préf., p. 83, où la même thèse est qualifiée de « docte sottise ». 
donc les lois et se constituait en «ennemi de l'État ${ }^{9}$ »; aussi la résistance n'avait-elle pour seul objet que le rétablissement de la paix authentique qui ne peut exister sans liberté. Les revendications de liberté font au contraire partie, aux yeux de Hobbes, des causes majeures des guerres civiles. Non seulement parce que la liberté (confusément conçue selon lui par ceux qui la revendiquent) fait l'objet d'une vénération dans les régimes populaires 10 , instables pour cette raison même, mais surtout parce que cette affection pour la liberté illimitée s'étaye sur une donnée anthropologique fondamentale : «l'amour» naturel de chaque homme « pour la domination d'autrui ». Or cet amour de la domination est incompatible avec la vie civile11, ce qui explique que la deuxième loi de nature conseille à l'individu d'abandonner son droit illimité sur toute chose ${ }^{12}$. Bien sûr, la «sûreté » que le représentant a pour fonction de procurer ne se réduit pas à la « simple préservation » et inclut aussi «toutes les autres satisfactions de cette vie $13 »$. Mais les libertés qui sont revendiquées contre l'arbitraire des gouvernants ne font justement pas partie de ces satisfactions, et sont même en fait incompatibles avec la jouissance de ces dernières.

Corrélativement, lorsque Hobbes veut rassurer son lecteur sur le fait que l'ensemble des droits des souverains d'institution rendent la "condition des sujets misérable », sa réponse est également dictée par la thèse selon laquelle les bienfaits de la paix civile, quelles qu'en soient les modalités, forment un bien tellement précieux qu'ils réduisent à rien les inconvénients qui l'accompagnent parfois: «La plus grande incommodité susceptible d'affliger le peuple en général, sous quelque forme de gouvernement que ce soit, est à peine sensible au regard des misères et des calamités affreuses qui accompagnent une guerre civile ou la condition déréglée d'hommes sans maîtres, non soumis aux lois ni à un pouvoir coercitif14. » En même temps qu'elles leur font voir en grand les petits désagréments induits par la soumission à un pouvoir délié des lois 15 , les passions des

9. John Milton, The Tenure of Kings and Magistrates (1649), in Political writings, Cambridge, CUP, 1991, p. 28, qui reprend George Buchanan, A Dialogue on the Law of Kingship among the Scots (1579), R. A. Mason et M. S. Smith (éd.), Aldershot, Ashgate, 2004, pp. 152-155 (« ennemi public »). 10. L, XIX, p. 227.

11. L, XVII, p. 173

12. L, XIV, p. 129 ; EL, I, 15, 2, p. 184.

13. L, XXX, p. 357

14. L, XVIII, pp. 190, 191 (trad. modif.).

15. Hobbes va plus loin dans EL, II, 5, 2, p. 268 : ces prétendus inconvénients «ne sont rien du tout». 
hommes les rendent aveugles aux misères infinies que la «science morale et civile» révèle en décrivant l'horreur qui attend le genre humain replongé dans l'état de nature.

\section{l'association \\ 2. Le désir de liberté rationnelle au fondement de}

Sidney renverse point par point cette argumentation. Si la prémisse de son raisonnement est bien le désir naturel de la liberté, tout son propos consiste - contre l'argument théologique de Filmer, qui assimile le désir naturel de la liberté au péché originel, mais aussi contre la version sécularisée qu'en donne Hobbes - à nier que la liberté illimitée soit l'objet du désir naturel de l'homme. Pour Sidney, ce que visent Filmer, Hobbes et les absolutistes dans leur condamnation des revendications de liberté, ce n'est pas la liberté mais la licence - deux notions qualitativement distinctes qu'ils veulent confondre pour légitimer la servitude politique.

C'est à l'aide de la notion de «créature rationnelle » que Sidney explicite la distinction entre liberté et licence. Il existe bien sûr des «affections », «passions » et certains « vices » qui poussent l'homme à désirer une liberté illimitée. Ce désir ne doit pourtant pas être dit naturel, puisqu'il est «incompatible » tant «avec un gouvernement quelconque » "qu'avec le bien que l'homme désire naturellement pour lui-même ». Parce que la fondation des sociétés est volontaire et parce que l'existence d'une société rend impossible pour quiconque une liberté illimitée, il est absurde de dire que naturellement « les hommes désirent la liberté sans bornes » puisqu'ils l'ont 《 universellement » « limitée » en rentrant en société16.

Ce dispositif, qui met la liberté rationnelle au centre des préoccupations des individus qui cherchent à s'associer, permet en outre d'examiner à nouveaux frais la thèse selon laquelle la paix est la valeur sociale première. Énoncée sous cette forme, elle est ambiguë : il y a différents types de paix, et celle dont on jouit prétendument sous les gouvernements tyranniques est la pire des conditions, car c'est la pire des misères que d'être soumis au pouvoir arbitraire d'un individu 17.

16. D, II, 20, pp. 191-192.

17. D, III, 5, p. 344 ; voir aussi D, II, 14, p. 154 et II, 15, p. 160. 


\section{Peuple et multitude (1)}

\section{Hobbes : l'objection sémantique}

Hobbes ne peut cependant se contenter de prétendre qu'il est contradictoire de vouloir détruire l'unique instrument de sa propre préservation : ce ne sont pas seulement les passions ou les affections de l'homme, mais aussi sa raison, qui ne disposent pas à la paix ${ }^{18}$. Aussi développe-t-il un argument bien plus puissant destiné à priver de signification l'idée même que le peuple pourrait se retourner contre le pouvoir.

La distinction entre multitude et peuple est au cœur de cet argument. En effet, dit-il, « les controverses qui surviennent au sujet du droit du peuple » proviennent de l'équivoque du mot «peuple », « qui a deux significations ». Selon le premier sens, le peuple n'est qu'une «multitude dissoute », c'est-à-dire une simple multiplicité d'individus dispersés et séparés les uns des autres. En parlant du droit du peuple en ce sens, on ne peut donc vouloir parler que du droit de chaque individu composant la multitude ; or «là où tout homme possède un droit distinct, il n'y a rien à quoi la multitude puisse avoir droit ». En outre, on ne saurait dire dans ce cas que les individus ont le droit de résister au magistrat, car là où « tout homme possède un droit distinct », on ne se trouve pas dans une condition civile, mais dans l'état de nature19.

Selon le second sens, le peuple est une «personne civile, c'est-à-dire soit un homme unique soit un conseil unique dans la volonté duquel est incluse et impliquée la volonté de chaque homme en particulier». S'il y a bien un magistrat - le souverain - et un peuple constitué, l'idée d'un droit de résistance du peuple n'en est pas moins dénuée de sens. En vertu de la logique d'inclusion des volontés particulières dans la volonté de la personne civile, il est en effet impossible que le peuple fasse valoir ses droits contre le souverain : les droits du peuple ne lui «appartiennent» qu'en tant qu'il est «contenu dans le corps de la république ou de la souveraineté $20 »$. Une fois le peuple constitué, les individus particuliers qui composaient la

18. Voir DC, V, 5, pp. 160-161 sur les traits humains (sens de l'honneur, appétits divergents, possession de la raison) qui portent à l'insurrection.

19. EL, II, 2, 11, pp. 248-249 ; voir aussi DC, VI, 1, p. 166.

20. EL, II, 2, 11, pp. 248-249 (trad. modif.); encore très présente dans DC (VI, 14 , p. 178 ; VI, 15 , p. 179 , VII, 7, p. 193 ; X, 5, p. 223 ; XII, 4, p. 244) pour établir la même thèse, cette logique de l'inclusion y cohabite avec celle de la lieutenance (DC, II, 5, p. 162). Toutes deux laisseront la place à la logique de la représentation dans L, XVII, p. 177. 
multitude ne possèdent plus de droits qu'ils pourraient légitimement réclamer contre le souverain, dans la mesure où ce dernier s'est approprié leurs «revendications et justifications $21 »$.

Le droit de résistance du peuple est donc un non-sens : d'une part, contrairement à ce que supposent les monarchomaques, le peuple ne saurait être par nature une entité à part entière - une universitas - capable d'établir des gouvernants et de les démettre22. Dans la nature, seuls existent des individus séparés les uns des autres. D'autre part, il n'est pas moins erroné de prétendre, de façon plus radicale encore, que ce n'est pas seulement «le peuple entier » qui possède le droit de résistance mais aussi les «individus » qui le composent23. Car une fois la société formée, les individus n'ont plus le droit de revendiquer quoi que ce soit contre le souverain.

$\mathrm{Au}$ demeurant, cette thèse est simplement le corrélat nécessaire du mécanisme mis en place plus tôt par Hobbes : l'acte de naissance du corps politique tient précisément au fait que chacun s'est « défai[t] ou dessais[i] de son propre droit de résister au bénéficiaire du transfert 24 ». La «non-résistance des membres est l'essence du pouvoir d'un corps politique », insiste Hobbes : «Quelle nation ou quelle république y a-t-il, où cet homme ou ce conseil, qui est virtuellement le tout, n'ait pas pouvoir absolu sur chaque membre particulier 25 ? »

\section{La réponse de Sidney : le peuple comme multitude}

Sidney défend la thèse strictement inverse. Le droit de résistance des individus qui constituent le peuple est au fondement de l'ordre juridique : «Toutes les lois doivent s'écrouler, les sociétés humaines qui subsistent par elles dissoutes $[\ldots]$, si les hommes ne peuvent pas légitimement se

21. EL, II, 2, 11, p. 249.

22. Voir EL, II, 8,9 , p. 321. C'est notamment la position défendue dans les Vindiciae contra tyrannos (1579), Amsterdam, 1610, II, p. 35.

23. Voir George Buchanan, A Dialogue on the Law of Kingship among the Scots, op. cit., pp. 152-153 ( $"$ ius est non modo universo populo, sed singulis etiam »).

24. EL, I, 19, 10, p. 224 ; voir aussi II, 1, 7, p. 230 ; DC, V, 7, p. 162 énonce la même thèse.

25. EL, II, 1, 18, p. 239. Hobbes affirme pourtant l'existence d'un droit individuel de résister à la violence (voir EL, I, 17, 2, p. 202; DC, I, 2, 18, p. 118 ; L, XIV, p. 132) qui est à la fois au cœur du contrat et totalement incompatible avec les droits nécessairement attachés à la souveraineté issue de ce même contrat. Pour intrigante qu'elle soit, on laissera de côté cette contradiction. 
défendre contre l'injustice par leur propre droit naturel, quand les voies prescrites par l'autorité publique ne peuvent être suivies $^{26}$. $\gg$ Les individus qui s'associent n'abandonnent donc pas le droit naturel par lequel ils établissent un gouvernement. Sidney dit être d'accord, contre Filmer, avec « ceux qui placent le pouvoir dans la multitude, et entendent par là une multitude composée d'hommes libres (multitude composed of freemen) qui pensent être dans leur intérêt de se rassembler (their convenience to join together) et d'établir les lois et les règles qu'ils s'obligent eux-mêmes à observer27.

Or, en dépit des similitudes avec Hobbes, on voit immédiatement qu'une telle conception de l'origine de la société affirme ce que Hobbes entendait réfuter : c'est bien la multitude, et non un souverain qu'elle aurait désigné, qui établit les lois, et ce n'est pas (ou du moins pas principalement) la crainte d'un châtiment qui fait que les hommes y obéissent 28 . En outre, quand les multitudes établissent des gouvernants, elles ne lui remettent pas leur droit naturel à «la liberté de se gouverner elles-mêmes 29 ». L'acte par lequel le gouvernement est établi n'est pas un abandon du droit naturel, mais un mandat conditionnel qui modifie les conditions d'exercice de ce droit sans le supprimer. La diversité des formes politiques observables «prouve clairement» que les «multitudes de créatures raisonnables [...] ont perpétuellement continué d'exercer ce droit (perpetually continued in the exercise of that right) 》 de déterminer elles-mêmes la forme de leur gouvernement, et de choisir ceux qui en ont la charge 30 .

Ainsi, dire que c'est par un «pouvoir qui leur est inhérent » que les multitudes ont eu des rois avant d'établir des lois pour y soumettre ces derniers, c'est dire qu'elles n'ont jamais aliéné ce pouvoir: "leurs libertés étaient naturellement inhérentes en eux-mêmes [i.e., les membres de ces multitudes], et leurs lois en étaient le produit ». Si les lois sont à la fois

26. D, III, 4, p. 340 .

27. D, II, 5, pp. 97-98; voir D, II, 1, p. 78.

28. Sidney articule de façon cohérente la nécessité d'un pouvoir coercitif (D, II, 24, p. 227) et la capacité naturelle de l'homme d'agir par devoir en respectant la « règle de la justice » «fondée sur un principe éternel de la raison et de la vérité » (D, III, 11, p. 381).

29. D, II, 5, p. 100.

30. D, II, 6, p. 112 (c'est nous qui soulignons) ; l'expression "créatures rationnelles» (ou raisonnables) revient en I, 6 (p. 21), I, 17 (p. 54) et I, 18 (p. 60). Voir John Locke, Second traité, $\S \$ 12,98,131,163-164$ et 230, où il utilise l'expression «créatures rationnelles » (sans parler de multitude) dans un sens très proche. 
postérieures à la création des rois et le produit de la liberté de la multitude, c'est que cette dernière a continué de jouir de sa liberté après l'établissement des rois. C'est donc le "même droit» qui établit le roi et le destitue, qui crée les lois et les modifie 31

\section{Une difficulté épistémologique}

Hobbes reconnaît au demeurant volontiers que la distinction qu'il établit entre peuple et multitude soulève une difficulté épistémologique, et il met en valeur son importance en soulignant qu'elle engage directement la stabilité de l'État ${ }^{32}$. Il mentionne notamment la tendance fréquente à croire que c'est le nombre qui fait le peuple, tendance que Jules César sut exploiter : il avait compris qu' « une armée est une telle force et une telle multitude qu'on peut aisément la persuader qu'ils [i.e., les soldats] sont le peuple 33 ». La vraie difficulté est ailleurs, toutefois, car la même confusion règne lorsque les individus sont comparativement peu nombreux ${ }^{34}$. La mise en garde épistémologique est particulièrement nette dans le Léviathan: «Les hommes ne parviennent pas à distinguer sans beaucoup d'étude et d'entendement une action unique de nombreux hommes (one action of many men) et de nombreuses actions d'une multitude (many actions of one multitude) [...] et sont par conséquent disposés à prendre pour une action du peuple ce qui est une multitude $d$ 'actions accomplies par une multitude d'hommes, conduits peut-être par l'effet de la persuasion d'un seul. »

Et c'est à nouveau l'exemple du tyrannicide qu'il choisit d'intercaler entre ce constat et cette conclusion, insistant ainsi sur les effets politiques de l'ignorance de la distinction, difficile à saisir par le commun, entre la légitimité d'un acte émanant d'une instance souveraine (le Sénat condamnant Catilina) et l'illégitimité d'actions individuelles de fait coordonnées (l'assassinat de César par quelques sénateurs) ${ }^{35}$.

Ainsi que le fait sentir la description des deux objets à ne pas confondre - « une action unique de nombreux hommes » et « de nombreuses actions d'une multitude » - le problème vient

31. D, III, 25, p. 457 ; voir D, II, 5, pp. 104-105, où Sidney continue de parler de multitude alors que la société est fondée.

32. Voir EL, II, 1, 2, p. 226 ; DC, VI, 1, p. 166 et XII, 8, pp. 247-248.

33. L, XXIX, p. 354 (c'est nous qui soulignons) ; voir aussi L, XVII, pp. 174-

175 ; EL, II, 1, 11, p. 249 ; DC, VI, 1, p. 166.

34. Voir DC, VI, 1, p. 168, et EL, II, 2, 11, p. 248.

35. L, XI, p. 100. 
surtout de la difficulté à saisir une différence ontologique: qu'est-ce qui différencie l'unicité d'une action accomplie par un groupe d'individus de la pluralité coordonnée d'actions différentes accomplies conjointement par plusieurs individus?

\section{Peuple et multitude (2) : l'enjeu ontologique de l'unité}

\section{Hobbes : l'unité réelle comme condition d'existence d'un peuple}

Quoiqu'elles diffèrent à des égards importants, toutes les versions que Hobbes propose de la distinction entre peuple et multitude font du critère de l'unité du peuple la condition nécessaire de son existence : pour être un peuple, il faut être un peuple. C'est probablement le De cive qui formule le plus nettement ce critère. Les individus qui se regroupent « ne sont pas quelque chose d'un», mais «plusieurs hommes", et la multitude n'est que le nom de ces hommes. Elle n'est donc pas une entité propre et distincte. Comme il le précise dans la note: «Une multitude, parce qu'il s'agit d'un mot collectif, s'entend comme signifiant plusieurs réalités (res plures): ainsi, une multitude d'hommes est la même chose que plusieurs hommes ${ }^{36}$. »

Or si elle n'est qu'une pluralité d'individus séparés, la multitude ne peut être considérée comme un agent à part entière. Rien de ce que peut faire un agent singulier ne lui est attribuable (promettre, contracter, transférer); rien de ce qui est imputable à une personne unique ne peut lui appartenir (ni bien, ni droit, ni action) ${ }^{37}$. La thèse du droit de résistance du peuple ne renvoie donc à rien dans la réalité. En revanche, les individus qui composent la multitude ne deviennent «quelque chose d'un », une « unité réelle », que par l'effet de la création d'un pouvoir commun auquel ils se soumettent 38 . Par quel mécanisme cette métamorphose se fait-elle?

L'existence d'un peuple suppose son unité, et cette dernière est impossible sans la soumission des volontés particulières à celle du souverain. Car c'est la multiplicité des volontés - le fait que chacun possède, exerce et agit en fonction

36. DC, VI, 1, pp. 166 , et 167 note (c'est nous qui soulignons «plusieurs réalités »)

37. DC, VI, 1, p. 166 (et p. 167 note) ; EL, II, 2, 11, p. 249.

38. Sur le processus de l'union, qui fait d'une multiplicité d'individus séparés (i.e., une multitude) une «personne civile» (un peuple), voir EL, I, 19, 8, p. 223 et II, 1, 1, p. 226. 
de « sa propre volonté », « son propre jugement 39 », « sa propre raison $40 »$, sa «propre conscience $41 »-$ qui conduit nécessairement à la dissension et, en définitive, à la guerre ${ }^{42}$. Les hommes étant toujours divisés dans leurs opinions sur « le meilleur usage et la meilleure application à faire de leur force 43 », le maintien de la paix ne peut dériver de la simple convergence des volontés particulières. Il faut donc substituer à cette trop fragile concorde entre des volontés distinctes une «volonté singulière » valant pour toutes. Or, cette volonté unique n'est à son tour possible que par l'assujettissement volontaire de chacun à cette « autre volonté [...], en sorte qu'elle tienne lieu de la volonté de tous et de chacun, quoi que veuille cet homme ou cette assemblée, concernant tout ce qui est nécessaire à la paix commune 44 ». L'union du peuple ne procède donc que de l'unité de la volonté singulière de la personne du souverain, autorisé par chacun : « on ne saurait concevoir l'unité dans une multitude sous une autre forme $45 »$.

\section{Sidney : La conception distributive du corps du peuple}

C'est en fait exactement ce que conteste Sidney, qui ne nie pas simplement que l'acte d'établissement de la société politique conduise à faire disparaître la multitude, mais encore que la multitude doive, pour agir, être une au sens où Hobbes l'entend, c'est-à-dire ne plus être une multitude d'individus mais devenir un peuple tel que «ni un citoyen, quel qu'il soit, ni tous les citoyens ensemble [...] ne doivent être considérés comme étant l'Etat 46 », c'est-à-dire le souverain.

Son souci de ne pas distinguer le peuple de la multitude est le plus net dans les passages mêmes ou Sidney utilise la notion de «corps du peuple». Mais peut-il y recourir pour le distinguer des individus particuliers sans remettre en question la cohérence de sa thèse selon laquelle la pluralité des membres de la multitude demeure après la fondation de la société civile ? La réponse est positive. Il distingue le corps du peuple comme entité des individus pris séparément dans un passage où il décrit leurs rapports respectifs d'obéissance vis-à-vis des gouvernants :

39. DC, VI, 1, p. 166

40. DC, V, 5, pp. 160-161.

41. DC, XII, 2, pp. 248-249.

42. Voir EL, II, 1, 2, pp. 226-227.

43. L, XVII, p. 175.

44. DC, V, 5-6, pp. 160-162 ; voir DC, VI, 1, p. 167 note.

45. L, XVI, p. 166.

46. DC, V, 9, p. 163 
« chaque homme privé considéré séparément (every private man singly taken) est soumis aux ordres du magistrat », et il n'est donc pas question de nier que les individus sont tenus d'obéir. En revanche, le «corps entier du peuple ne l'est pas (is not so) » : parce qu'il est le « maître » des lois et soumet le magistrat à ces dernières, le peuple n'est en rien lié vis-à-vis du magistrat 47 .

Le singulier du « corps entier du peuple »n'indique-t-il tout de même pas que Sidney vise par cette expression un être collectif, c'est-à-dire une entité différente de l'ensemble distributif des membres du peuple? S'il ne développe pas d'argument infirmant directement cette lecture, un indice textuel permet semble-t-il de le faire. Sidney poursuit son raisonnement en soulignant que la soumission des individus est conditionnelle : l'obéissance que l'individu doit au magistrat est «mesurée par la loi générale », c'est-à-dire le salut du peuple, conçu par Sidney de façon explicitement distributive 48 . Mais au lieu de poursuivre la description de ce qui distingue «chaque homme privé » du « corps entier du peuple », il en conclut à leur identité : «par conséquent, le corps entier d'une nation (whole body of a nation) ne saurait être tenu à aucune autre obéissance que celle qui, selon leur propre jugement (their own judgement), est compatible avec le bien commun 49 ». La thèse de l'obéissance conditionnelle autorise donc à assimiler le tout et l'ensemble des parties qui le composent ${ }^{50}$. Cette assimilation est requise si l'on veut maintenir que «tout homme a un droit de résister d'une manière ou d'une autre à ce qu'on ne devrait pas lui faire $51 »$, et elle explique pourquoi Sidney continue de décrire le « corps tout entier (compleat body)» ou le « corps civil » du peuple comme une multitude52.

Difficile d'ignorer de façon plus ostensible l'effort de Hobbes pour mettre en cause pareille identification. Hobbes, bien sûr, aurait vu dans le développement de Sidney un propos aussi confus que dangereux. Mais il n'y a de confusion que si l'on refuse de considérer, avec Hobbes, que des volontés humaines s'accordant « de façon solide» sur des finalités

47. D, III, 36, p. 519

48. D, III, 36, p. 519 ; sur le salut du peuple entendu distributivement, voir Ch. Hamel, L'esprit républicain: droits naturels et vertu civique chez Algernon Sidney, Paris, CLassiques Garnier, 2011, pp. 243-248.

49. D, III, 36, p. 519.

50. Voir D, III, 13, p. 391, pour un mouvement parfaitement identique.

51. D, III, 4, p. 339.

52. Voir D, II, 5 , p. 99 , et II, 1 , p. 78 . 
puissent être autre chose qu'une « multitude dissoute 53 ». Sidney échappe à la contradiction construite par Hobbes précisément parce qu'il refuse que l'unité du peuple par la réduction à l'unicité de la volonté d'un homme (ou d'une assemblée) soit la condition qui rende possible son existence même en tant que peuple.

S'il le refuse, c'est bien sûr parce qu'il adopte une anthropologie rationaliste où un certain nombre de «notions communes » ou de principes sont des vérités évidentes par la seule «lumière de la raison » ou du «sens commun 54 ». Mais c'est aussi parce que, en vertu de ces mêmes principes, les conséquences de la théorie de l'unité du peuple sont inacceptables.

\section{À quel prix devient-on un peuple ? Les conséquences outrées de la thèse hobbesienne}

\section{La très paradoxale souveraineté du peuple}

La conception du processus par lequel une multitude d'individus devient un peuple entraîne chez Hobbes des conséquences dont le caractère outré révèle la nature problématique des prémisses dont elles découlent. Pour le comprendre, partons du «paradoxe »- reconnu comme tel par Hobbes dans le souci de rassurer les fauteurs d'anarchie partisans des régimes populaires et de la souveraineté du peuple - selon lequel « en tout État, le peuple règne $[\ldots]$ : le peuple est souverain, même dans les monarchies », où « le roi est le peuple ». L'unicité de la volonté, qui conditionne l'existence même d'un agent, implique que la volonté du prince tienne lieu de volonté du peuple : «le peuple veut par la volonté d'un seul homme » et ne peut vouloir que par elle, parce qu'il n'est que par lui. Le «sens » auquel on peut dire que les citoyens commandent souverainement est donc très particulier et fort paradoxal. Parce que la médiation par la volonté du souverain est constitutive de l'existence du peuple, les citoyens ne sont souverains qu'au sens où ils ne peuvent pas exercer la souveraineté ou, plus précisément, qu'en tant qu'ils sont dépossédés de la souveraineté et doivent la subir comme « sujets » sans murmurer ${ }^{55}$.

53. EL, II, 1, 11, pp. 248-249, et II, 1, 2, p. 227.

54. D, I, 2, pp. 8, 11 ; II, 8, p. 123 ; II, 30, p. 291 ; I, 3, p. 13.

55. DC, XII, 8, p. 248, où se trouvent toutes les citations de ce paragraphe (c'est nous qui soulignons). 
On conçoit, pour reprendre l'expression de Hobbes, qu'on puisse voir là un «paradoxe», et que «les hommes du commun» (la multitude ignorante ?) « et d'autres» (les théoriciens du droit de résistance) «ne remarquent absolument pas » en quel sens le peuple règne toujours 56. Mais le paradoxe selon lequel un peuple n'est souverain qu'en tant qu'il est incapable d' "agir » autrement que par la volonté d'un individu ou d'une assemblée devant être considérée comme la volonté de chacun des individus qui forment la multitude n'est-il pas une parfaite mystification?

\section{L'abandon du droit de se gouverner soi-même}

Revenons pour nous en convaincre à la condition d'existence d'un peuple : un peuple n'existe que s'il est doté d'une volonté unique, laquelle n'est créée que par l'effet de l'abandon par chacun de son droit de s'autogouverner.

Le seul moyen d'ériger un pouvoir qui permette aux hommes de «vivre satisfaits (contentedly) » est de «conférer tout leur pouvoir et toute leur force (all their power and strength) à un seul homme ou une seule assemblée qui puisse réduire toutes leurs volontés [...] en une seule volonté ». Hobbes poursuit immédiatement en explicitant le sens de ce transfert à l'aide de sa théorie de l'autorisation : "Cela revient à dire : désigner un homme ou une assemblée pour endosser leur personne, et que chacun s'avoue et se reconnaisse lui-même comme l'auteur de tout ce qu'aura fait ou fait faire, quant aux choses qui concernent la paix et la sécurité commune, celui qui a ainsi endossé leur personne57.» Ce processus d'autorisation n'est pas un engagement à donner son accord à ce que fait le souverain, car cela supposerait que chacun conserve son jugement. Or désigner un représentant pour «endosser notre personne », ce n'est pas seulement «soumettre sa volonté et son jugement à la volonté et au jugement » du souverain, c'est les lui abandonner, comme le pose de façon univoque l'acte de langage hypothétique par lequel le souverain est créé : «J'autorise cet homme ou cette assemblée, et je lui abandonne mon droit de me gouverner moi-même (give up my right of governing myself), à cette condition que tu lui abandonnes ton droit et que tu autorises toutes ses actions de la même manière ${ }^{58}$. »

56. DC, XII, 8, p. 248.

57. L, XVII, p. 177 (trad. modifiée ; c'est nous qui soulignons).

58. L, XVII, p. 177. Voir les textes parallèles dans DC, V, 6-9, pp. 160-163;

EL, I, 19, 10, p. 224 ; EL, I, 15, 3, p. 185 ; EL, II, 5, 2, p. 269. 
C'est cet abandon qui permet d'expliquer que «l'unité réelle » de la «seule et même personne »créée est bien plus qu'un simple «consensus 59 ». Abandonner son droit de se gouverner, c'est donc bien abandonner sa capacité de juger des conditions propres à réaliser ou maintenir la paix : comme l'énonce la description de «l'essence de la république », c'est désormais la «personne unique» issue de la convention qui « use de la force et des ressources de tous comme elle le jugera expédient en vue de la paix et de leur défense commune60 ».

Si tel est bien le seul moyen de créer une république, il faut alors conclure que la vie civile est la négation même de l'autogouvernement, et que le bonheur temporel de l'homme suppose le renoncement par chacun au pouvoir d'en organiser collectivement la jouissance.

\section{La réponse de Sidney}

À partir de l'expression de multitude de créatures rationnelles, Sidney élabore un raisonnement exactement opposé. Étant donné que «le pouvoir est à l'origine dans la multitude » et que les individus sont naturellement égaux, « il est impossible de croire que des créatures rationnelles (rational creatures) aient avancé l'un ou quelques-uns de leurs égaux sinon en considérant leur propre bien ». Et c'est seulement parce que les individus qui composent la multitude sont des « créatures rationnelles », dotées des capacités minimales permettant de juger correctement des affaires publiques, que Sidney ajoute ne pas voir «d'inconvénient à laisser aux multitudes », une fois le gouvernant choisi, « le droit de juger (right of judging) si cela est dûment accompli ou non $61 »$. Ce qui ne signifie pas que tous les individus aient les aptitudes requises pour gouverner de façon juste et efficace, mais seulement que toute créature rationnelle possède naturellement un «sens du droit » qui lui permet de sentir et d'évaluer de façon correcte l'action politique conduite par les gouvernants. Or, parce que les individus cherchent « leur propre bien » dans l'association et que celle-ci n'est faite que pour le réaliser, ils sont «les seuls à savoir si la fin pour laquelle ces magistrats ont été créés est accomplie ou non $62 »$.

59. L, XVII, p. 177 ; EL, I, 12, 7-8, pp. 167-168 ; I, 19, 6, p. 222.

60. L, XVII, p. 178 (c'est nous qui soulignons) ; voir aussi L, XVIII, p. 179, et L, XVI, pp. 166-167.

61. D, I, 6, p. 21 (c'est nous qui soulignons).

62. D, III, 42, p. 549-50 (c'est nous qui soulignons). 
Certes, cette thèse ne peut être démontrée, aux yeux de Sidney, car elle relève du «sens commun » le plus élémentaire : «Et si l'on doute au sujet de savoir qui est le juge le plus adéquat (fittest judge) pour déterminer la question, le sens commun nous informera que si le magistrat reçoit son pouvoir par élection ou donation, ceux qui l'élisent [...] savent le mieux (best know) si le bien qu'ils cherchaient est obtenu ou non [...]. Personne d'autre que le peuple, pour qui et par qui les constitutions sont faites, ne peut être juge dans de tels cas 63 . » Ce que l'on peut dire, simplement, c'est que ce pouvoir de juger et donc de choisir les représentants (ainsi que la forme du gouvernement) participe $\mathrm{du}$ «principe de liberté » qui caractérise la nature de l'homme.

C'est ce principe de liberté que récuse Filmer en prétendant que « rien de tout cela [i.e., les questions politiques] ne fut laissé au choix des hommes », et que ces derniers «ne doivent pas s'enquérir de ce qui les conduit à leur propre bien 64 ». Or, si la condition politique est certes pour Hobbes une création humaine, et donc l'effet d'un choix, en soutenant que les hommes n'y parviennent qu'en abandonnant leur capacité de jugement politique, il confère à cette décision l'allure d'un renoncement définitif à exercer toute forme de citoyenneté critique. Si telle est selon lui la condition pour jouir des satisfactions de la vie, la recherche des «principaux avantages de la vie dont nous jouissons » repose au contraire pour Sidney sur l'exercice libre de cette faculté d'examen et de choix : c'est parce que Dieu a donné à «tous les hommes » une «capacité de juger ce qui est bon pour eux-mêmes » qu'il a «accordé à tous de la même manière une liberté d'inventer» les formes politiques de leur choix 65 .

Les conséquences contre-intuitives auxquelles Hobbes parvient vont même plus loin : les processus de la représentation et de l'autorisation ne supposent pas seulement que chaque individu abandonne son droit de se gouverner lui-même, ils impliquent encore que chacun se reconnaisse positivement comme l'auteur de toutes les actions du souverain : «chacun donne à celui qui les représente tous l'autorité qui dépend de lui en particulier, reconnaissant pour siennes toutes les actions accomplies par le représentant (owning all the actions the representer doth)66 ${ }^{\prime}$.

63. D, III, 21, p. 440 (c'est nous qui soulignons).

64. D, I, 1, p. 6.

65. D, I, 6, p. 20.

66. L, XVI, p. 166 (c'est nous qui soulignons) ; voir aussi p. 163. 
Tout ce que le souverain dit faire pour protéger les individus est réputé être autorisé par les individus eux-mềmes ${ }^{67}$, ce qui signifie que je ne dois pas simplement considérer que ses actions sont nécessairement légitimes, mais plus exactement les revendiquer comme miennes et donc les soutenir de toutes les forces dont je dispose, puisque je dois m'en considérer l'auteur : «toute action [du souverain] [...] est à la fois l'acte de cet homme même [i.e., le souverain] et l'acte de tous les autres parce qu'il est accompli au nom et en vertu du droit de chacun d'eux en particulier (in the person and by the right of every one of them in particular) [...] les actions [du souverain] sont revendiquées (avouched) par tous, et exécutées par la vigueur de tous, unis dans le souverain $68 »$. La version latine est particulièrement éloquente : «quiconque lui [i.e., au souverain] fait grief d'une mauvaise gestion est soi-même auteur de celle-ci et s'accuse lui-même » de s'être porté préjudice à lui-même, ce qui est absurde 69 .

Le coût de telles conséquences est très élevé, et Hobbes l'assume complètement : étant donné ce que sont les hommes, toutes les autres formes d'union n'en sont pas vraiment; irrémédiablement instables, elles reconduisent en définitive les hommes à une condition où leur désir de sûreté ne peut être satisfait. Ceux qui ne sont pas prêts à accepter ces conséquences doivent réviser les prémisses dont elles procèdent : c'est ce que fait Sidney, en posant au principe de sa pensée politique l'intuition selon laquelle les hommes sont capables de déterminer par eux-mêmes les moyens de jouir de la liberté collectivement.

Le désaccord entre les êtres humains est-il tel qu'ils doivent, pour espérer vivre ensemble, abandonner leur capacité individuelle de juger des conditions les plus propres à assurer une coexistence pacifique? Au contraire, la prémisse selon

67. L, XVIII, p. 181.

68. L, XVIII, pp. 181, 182 (trad. modif.).

69. L, XVIII, p. 182 , note 15 ; et p. 183 . Il y a certes une limite à cette identification: bien que le souverain soit en droit de me donner la mort pour une infraction commise (L, XVIII, p. 187) je ne peux plus considérer comme mienne l'action par laquelle il s'en prend à mon existence (L, XIV, p. 132). Cette limite soulève assurément une difficulté interne à la théorie du droit du souverain; mais indépendamment même de cette difficulté, la position toute paradoxale de Hobbes est que la résistance que j'oppose alors au souverain n'est pas légitimée par l'injustice qu'il commet, puisque le souverain seul possède le droit de dire ce qu'est le juste et l'injuste. 
laquelle ils s'accordent sur les finalités de l'association supposet-elle que ce droit d'examen soit inaliénable ? La stabilité d'une société réglée par l'obéissance aux lois peut-elle supporter d'organiser institutionnellement la contestation et de rendre possible constitutionnellement l'insurrection ? On l'a vu, les réponses que Hobbes et Sidney apportent à ces questions sont absolument incompatibles. L'intérêt de les comparer est qu'elles dérivent toutes deux de l'individualisme.

À l'idéal du gouvernement du peuple par le peuple, les partisans de la monarchie et de l'aristocratie opposèrent systématiquement la thèse selon laquelle la multitude est fondamentalement dépourvue des facultés rationnelles qui rendraient l'autogouvernement possible. "L'horrible système » de Hobbes 70 tient précisément au fait qu'il systématise cette thèse en l'universalisant : c'est la nature même de l'homme qui fait du renoncement à sa faculté d'examiner les termes de la vie commune la condition indispensable et la forme nécessaire du pacte qui donne naissance à la société. Pour Sidney, au contraire, la faculté individuelle de jugement et d'examen est l'outil principal de construction, de rénovation et d'amélioration de la société que les hommes instituent pour jouir de leur liberté.

70. Rousseau, «Que l'état de guerre naît de l'état social», in Euvres complètes, op. cit., p. 602. 